\title{
Testing the stability of heavy dark matter with up-coming radio neutrino telescopes
}

\author{
Rasmi Hajjar ${ }^{a, *}$ \\ ${ }^{a}$ Scuola Superiore Meridionale, Università degli studi di Napoli "Federico II", \\ Largo San Marcellino 10, 80138 Napoli, Italy \\ E-mail: rasmienrique.hajjarmunoz@unina.it
}

Astrophysical neutrinos with energies higher than $E_{v}>10 \mathrm{PeV}$ have not been measured yet, therefore, we do not know what kind of sources are contributing to this high energy neutrino flux. Concretely, we are interested in testing the hypothesis in which we have a hidden contribution coming from the decay of heavy dark matter particles. The first neutrino radio telescopes will be constructed during the following decade, having as a principal goal the detection of the cosmogenic neutrinos, and being able to disentangle the principal source contributing at these high energies for the astrophysical neutrino flux. In this work we study the projected sensitivity of up-coming neutrino radio telescopes, such as RNO-G, GRAND and IceCube-Gen2 radio array, to decaying dark matter scenarios. We perform a forecast analysis in order to place conservative constraints on the lifetime of dark matter within the range $m_{\mathrm{DM}}=\left[10^{7}-10^{15}\right] \mathrm{GeV}$ after assuming the dominant astrophysical neutrino source, the one that will act as our background for the hidden dark matter signal. These forecasted limits open a new parameter space for some decaying channels, and complement the limits obtained for the $b$ channel due to its multi-messenger agreement.

$37^{\text {th }}$ International Cosmic Ray Conference (ICRC 2021)

July 12th - 23rd, 2021

Online - Berlin, Germany

\footnotetext{
${ }^{*}$ Presenter
} 


\section{Introduction}

During the past decades some evidence pointed out that the majority of the energy budget of the Universe is dominated by a dark sector that still remains hidden to us. Inside this dark sector we find one component, the dark matter (DM), that we have been able to see interacting via gravitational interaction, but it has evaded other kinds of direct detection methods that usually depend on the nature of this unknown component. Another direction in which we can search signatures from this dark matter component is the indirect detection via cosmic rays, gamma rays and neutrinos emitted from the decay or annihilation of such particles. A lot of effort has been made in this direction due to the experimental measurements of the cosmic ray and gamma ray asatrophysical fluxes. Using the neutrino messenger we find that IceCube $[1,2]$ measured astrophysical neutrinos and helped to complement the indirect multi-messenger searches. However, the energy window with $E_{v}>10 \mathrm{PeV}$ is observationally unexplored using neutrino observations, being a crucial energy range for the possible fluxes generated by heavy dark matter particles.

Neutrinos are the perfect messengers because contrary to cosmic rays and gamma rays they can travel unaffected by collisions with any foreground and since they are also neutral particles they are not deflected by any galactic or extragalactic magnetic field. Therefore we will be interested in measuring high energy neutrino fluxes and see if we could identify any unknown contribution that we could associate with dark matter. Up until now the most important measurements of the high energy neutrino flux come from the upper limits placed by PAO [3] and ANITA [4]. In this work, we are going to introduce three future planned neutrino radio telescopes that will be able to shed a light on the ultra-high energy neutrino flux. Their primary aim is to measure the neutrinos that are produced through the interaction of cosmic rays with the Cosmic Microwave Background (CMB) photons, the cosmogenic neutrinos [5]. This contribution is a guaranteed contribution although its magnitude is subject to severe theoretical uncertainties. Moreover we can also expect further neutrino contributions coming from hadronic production in certain astrophysical environments. Among all the possibilities we are going to focus only on the newborn pulsars [6] contribution due to the fact that it will leave the higher neutrino imprint on the telescopes under study. Therefore these neutrino telescopes will also be able to disentangle which one is the dominant source of the neutrino flux at these ultra-high energies.

In this work [7] we have used the future neutrino radio telescopes RNO-G [8], IceCubeGen2 radio array $[9,10]$ and GRAND [11] in order to explore the parameter space of heavy dark matter particles with masses in the range $\left[10^{7}-10^{15}\right] \mathrm{GeV}$, focusing on the decay rather than in the annihilation due to the higher produced neutrino fluxes. We will assume that the neutrino telescopes will measure only the cosmogenic neutrino contribution or the newborn pulsars one and extract in this way the limits that we could place on the lifetime of the dark matter particles using the projected sensitivities of the aforementioned telescopes. We will take into account the higher theoretical prediction of each astrophysical contribution. In this we are making a conservative choice and we will place conservative constraints due to the non-observation of a dark matter flux. 


\section{High energy neutrino fluxes}

Since we are going to test the decaying dark matter neutrino fluxes we need to make an assumption on the decay channel of this unknown particle. Here we assume that a DM particle can decay into a pair of standard model $(\mathrm{SM})$ particles $(\mathrm{DM} \rightarrow f \bar{f})$ with a $100 \%$ branching ratio. In particular we are going to focus on three different channels: $b, \tau$ and $v$. These are representative of hadronicphilic, leptophilic and neutrinophilic dark matter particles, respectively. Also, for the neutrino channel we take equipartition among neutrino flavours at production. Here we are going to assume the minimal decaying scenario, with only two parameters: the mass of the dark matter particles $\left(m_{\mathrm{DM}}\right)$ and the lifetime of them $\left(\tau_{\mathrm{DM}}\right)$.

Dark matter particles distributed along the galactic halo and disperse on the extragalactic medium give rise to neutrino fluxes that we can parametrize in different components: the galactic and the extragalactic one. The differential flux of neutrinos and antineutrinos per flavour $\alpha$ of the former one is

$$
\frac{\mathrm{d} \Phi_{v_{\alpha}+\bar{\nu}_{\alpha}}^{\text {gal. }}}{\mathrm{d} E_{\nu} \mathrm{d} \Omega}=\frac{1}{4 \pi m_{\mathrm{DM}} \tau_{\mathrm{DM}}} \frac{\mathrm{d} N_{\alpha}}{\mathrm{d} E_{v}} \int_{0}^{\infty} \mathrm{d} s \rho_{\mathrm{DM}}[r(s, \ell, b)] .
$$

Here the quantity $\rho_{\mathrm{DM}}$ is the dark matter galactic distribution, which we assumed to be a NavarroFrank-White profile [12], expressed in terms of the galactocentric radial coordinate $r$, which can be in turn expressed as a function of the galactic coordinates. The $d N_{\alpha} / d E_{v}$ quantity is the spectra of neutrinos generated from dark matter decay. In this work we are going to use the spectra obtained from the recent code HDMSpectra [13] We can also find on the above equation both dark matter parameters, mass and lifetime.

On the other hand the extragalactic component reads as

$$
\frac{\mathrm{d} \Phi_{v_{\alpha}+\bar{\nu}_{\alpha}}^{\text {ext.gal. }}}{\mathrm{d} E_{\nu} \mathrm{d} \Omega}=\left.\frac{\Omega_{\mathrm{DM}} \rho_{c}}{4 \pi m_{\mathrm{DM}} \tau_{\mathrm{DM}}} \int_{0}^{\infty} \frac{\mathrm{d} z}{H(z)} \frac{\mathrm{d} N_{\alpha}}{\mathrm{d} E_{\nu}^{\prime}}\right|_{E_{\nu}^{\prime}=E_{\nu}(1+z)},
$$

where $\rho_{c}$ is the critical density of the Universe, $\Omega_{\mathrm{DM}}$ encodes the density of the DM particles in the Universe, $z$ is the cosmological redshift and $H(z)$ is the Hubble expansion rate taking into account the latest Planck measurements of the cosmological parameters [14]. We remark that opposite to gamma rays, neutrino absorption in the intergalactic medium is negligible.

Since generally the information that we can extract from other works is expressed summed over all neutrino flavours we will also work under this conditions in order to preserve the consistency of the analysis. Moreover, we will also consider angle averaged fluxes because the experiments do not provide the sensitivity as a function of arrival direction. Therefore, the DM fluxes that we are going to work with will be

$$
\frac{\mathrm{d} \Phi_{3 \nu}^{\mathrm{DM}}}{\mathrm{d} E_{\nu}}=\sum_{\alpha} \int \mathrm{d} \Omega\left[\frac{\mathrm{d} \Phi_{v_{\alpha}+\bar{v}_{\alpha}}^{\text {gal. }}}{\mathrm{d} E_{\nu} \mathrm{d} \Omega}+\frac{\mathrm{d} \Phi_{v_{\alpha}+\bar{\nu}_{\alpha}}^{\text {ext.gal. }}}{\mathrm{d} E_{\nu} \mathrm{d} \Omega}\right] .
$$

We show in Fig. 1 the ultra-high energy astrophysical neutrino scenario. Together with the higher energy measurements of IceCube HESE events we show the astrophysical predicted fluxes: cosmogenic and newborn pulsars. In both cases we have shown the higher limit of the predicted 


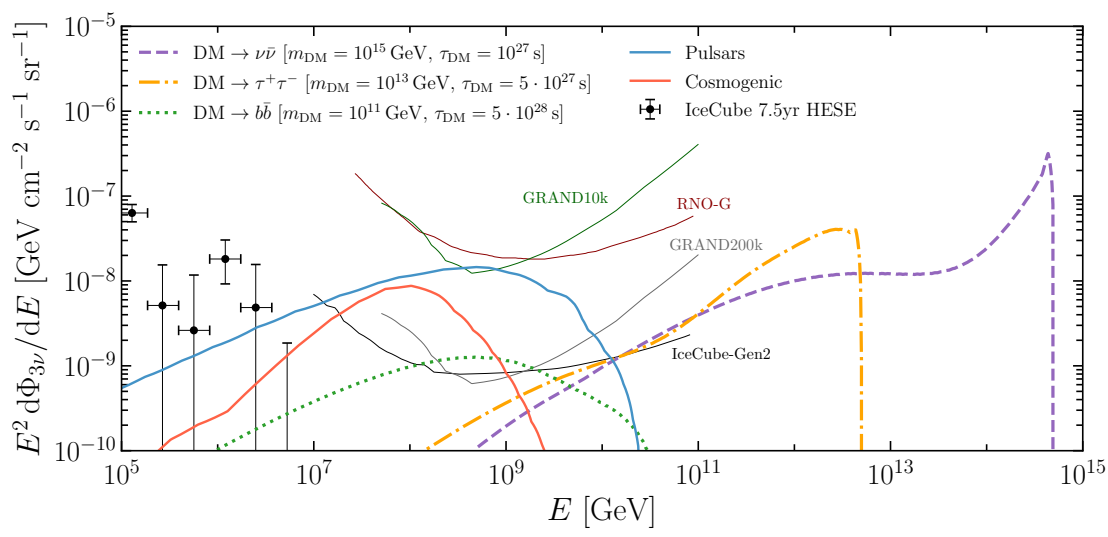

Figure 1: All-flavour neutrino fluxes predicted in two astrophysical scenarios (red line for cosmogenic neutrinos and blue line for newborn pulsar neutrinos) and in three different benchmark cases of decaying dark matter. The thin lines show the 3-year sensitivity reach of upcoming neutrino radio telescopes, while the data points correspond to the 7.5 year IceCube HESE sample [2].

fluxes due to the fact that this will draw the most conservative results. This is because in this case these astrophysical fluxes will be the ones that we assume to measure, and a higher number of observed events will be able to hide a higher dark matter contribution, obtained with a lower lifetime of the dark matter for each particular decay channel. We also show three benchmark cases for the DM fluxes obtained using HDMSpectra in order to depict the shape of the different channels. Finally, the sensitivities of the different neutrino telescopes are also shown in the plot in order to see clearly the reach of the aforementioned telescopes. In GRAND we show two different configurations, one with 10.000 antennas (GRAND10k) and the final one with 200.000 (GRAND200k).

From these fluxes we can obtain the different number of events that we are going to take into account for our analysis: the number of events that we will expect from astrophysical sources and the ones coming from dark matter. It is important to remark that the number of astrophysical neutrino events will act as our source of background, and the expected detector background is negligible, as the collaboration report on their respective design reports. This detector background numbers have been validated by ARA, which has achieved a background of 0.01 events in two stations over 1100 days [15].

In the method that we are going to follow we will obtain the number of expected events coming from the astrophysical source of our choice and then we will see for each channel which is the lifetime of the dark matter that predict a flux whose number of events allow us to exclude the dark matter presence with $95 \%$ confidence level. In this way we can clearly see how choosing the higher expected astrophysical fluxes will lead to lower constraints: a higher flux will predict more number of events and therefore we will need a lower lifetime for the dark matter in order to produce higher neutrino fluxes and obtain the confidence level of our choice.

\section{Forecasted lifetime limits of heavy decaying dark matter}

The main results of the work are contained in Fig. 2, where the most probable lower limits that would be extracted from the future neutrino telescopes are shown together with their $2 \sigma$ bands. 

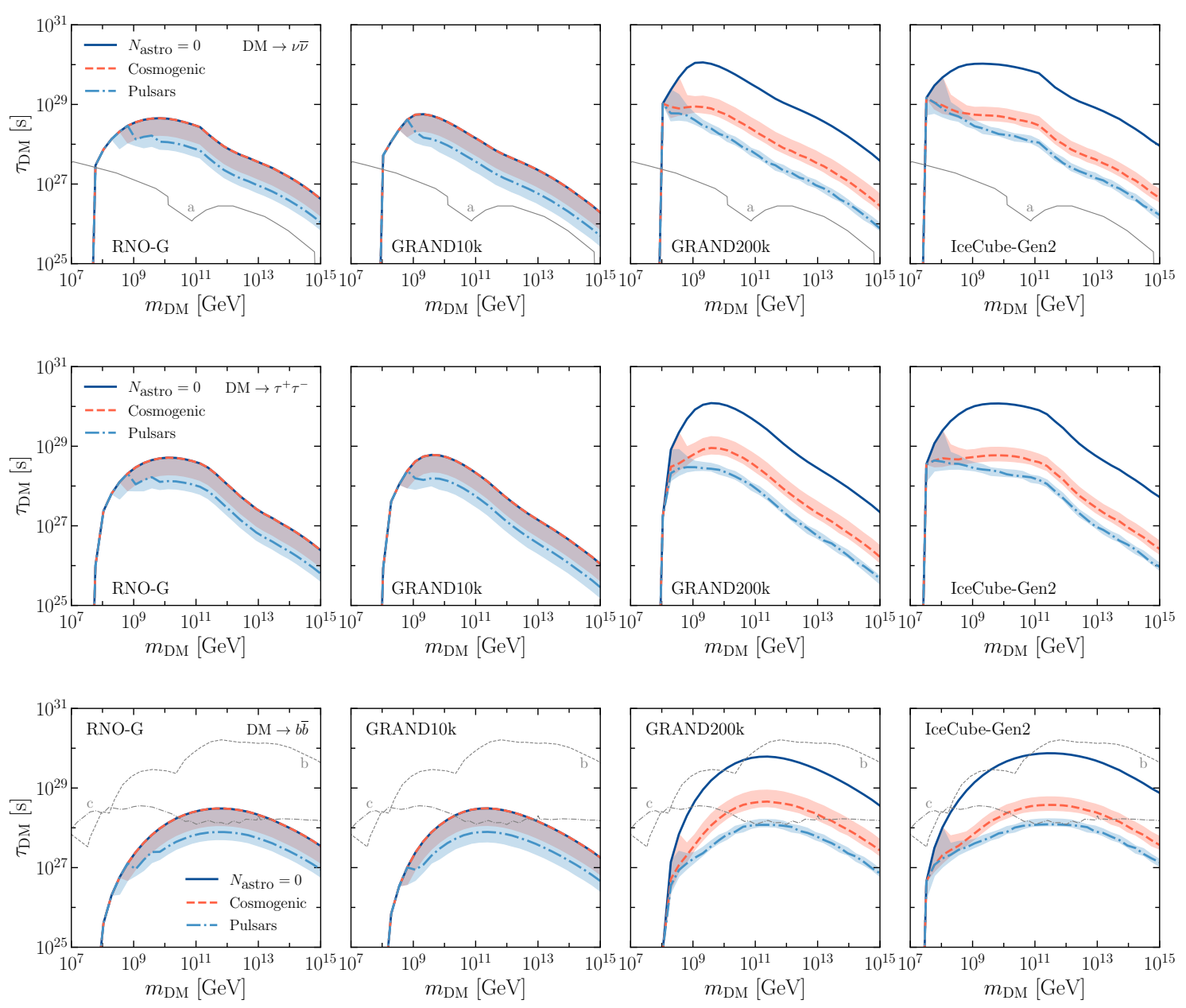

Figure 2: Projected 3-year constraints at $95 \% \mathrm{CL}$ in the plane $m_{\mathrm{DM}^{-}} \tau_{\mathrm{DM}}$. Each row corresponds to a different DM decay channel, while each column to a different upcoming neutrino radio telescope. The bands represent the $2 \sigma$ intervals according to the Poisson distribution in Eq. (??) of observing a given number of events in the cosmogenic (red color) and the newborn pulsars (blue color) scenario. The red dashed and blue dot-dashed lines show the most probable constraints on $\tau_{\mathrm{DM}}$ assuming the observation of astrophysical neutrinos. The dark-blue solid lines display the limits obtained in the case of zero detected events $\left(N_{\text {astro }}=0\right)$. The thin gray lines are the existing constraints in the literature: a) for the $v \bar{v}$ channel with neutrino data from IceCube, PAO and ANITA [16]; b) for the $b \bar{b}$ channel with galactic multimessenger data [17]; c) for the $b \bar{b}$ channel with extragalactic multimessenger data [17].

In the figure we show the different combinations of channel, telescope and expected astrophysical background. Here we include also the possibility of not having any astrophysical background at our detectors, a possible scenario due to the fact that we do not know the magnitude of the cosmogenic flux nor if there are other neutrino sources (like the newborn pulsars) contributing at these high energies. In the plot there are also shown the existing constraints placed on the different decay channels by other detectors, mainly due to gamma-ray measurements for the $b$ channel and due to IceCube, PAO and ANITA [16] on the $v$ one.

We can see that the cosmogenic constraints lead to higher limits than the newborn pulsars 
case. This stems from the fact that the number of expected observed events is lower in the case of cosmogenic neutrinos than the newborn pulsar ones. Therefore, a higher number of expected detected events will lead to lower lifetime constraints since we are testing only upper-fluctuations. As we can see the stronger constraints will be obtained in the case where the telescopes do not detect any neutrino. Moreover, the three cases converge for the lower DM masses, an expected behavior since we have an expected number of events close to 0 because these masses are close to the lower energy limit that the telescopes can detect. In fact, the sharp cutoff at lower masses is a consequence of reaching this lower energy limit of the telescopes effective areas. Finally, in the smaller detectors (RNO-G and GRAND100k) we have that the cosmogenic expected value and the $N_{\text {astro }}=0$ cases coincide, since the cosmogenic flux is much lower than the reach of the neutrino telescopes, as we can clearly see on Fig. 1.

Before concluding we want to comment the effect that the inclusion of an additional detector background component on the experiments. We have performed the analysis using the low expected values reported at each telescope design report and we found that our constraints weaken something between $\sim 10 \%$ and $\sim 30 \%$ for the newborn pulsars and cosmogenic astrophysical contributions respectively, being all values contained between our $2 \sigma$ bands.

\section{Conclusions}

The future of the high energy neutrino astronomy will include the valuable contribution of the radio neutrino telescopes, which will be built upon the next decade. In particular, we have focused on four benchmark telescopes: RNO-G, GRAND10k, GRAND200k and IceCube-Gen2 radio array. These telescopes will try to detect the cosmogenic neutrinos and will shed a light on the different sources that produce neutrinos at these high energies. Moreover, they will allow to study the heavy decaying dark matter parameter space using neutrino observations. In order to do this we have forecasted the performance of these neutrino telescopes by assuming different astrophysical sources generating the neutrino flux at high energies. The dark matter fluxes have been generated assuming the minimal decaying dark matter scenario for the hadronicphilic, leptophilic and neutrinophilic dark matter particles.

Our results are robust and conservative since they have been obtained analyzing the total neutrino events without considering their angular and energy distribution. Our projections show that the limits that these telescopes will be able to place could open a new parameter space for the different decaying channels (in particular the $v$ and $\tau$ ones), or at least will be a good complement to the current existing ones, obtaining a multi-messenger agreement. As we can see the $b$ channel all of the parameter space that neutrino telescopes can probe is disfavoured by cosmic-ray and gamma-ray data, but indirect dark matter searches with neutrinos are highly complementary to the ones with the other messengers due to different theoretical and experimental uncertainties.

For a more detailed explanation of the analysis performed we refer the reader to [7], where more information about the methods used could be found. 


\section{Acknowledgements}

I thank Marco Chianese for useful comments. I acknowledge the research grant number 2017W4HA7S "NAT-NET: Neutrino and Astroparticle Theory Network" under the program PRIN 2017 funded by the Italian Ministero dell'Università e della Ricerca (MUR), that covered the costs to attend the ICRC 2021 conference.

\section{References}

[1] M. G. Aartsen et al. [IceCube], Science 342 (2013), 1242856 doi:10.1126/science.1242856 [arXiv:1311.5238 [astro-ph.HE]].

[2] R. Abbasi et al. [IceCube], [arXiv:2011.03545 [astro-ph.HE]].

[3] D. Veberic [Pierre Auger], [arXiv:1708.06592 [astro-ph.HE]].

[4] P. W. Gorham et al. [ANITA], Phys. Rev. D 99 (2019) no.12, 122001 doi:10.1103/PhysRevD.99.122001 [arXiv:1902.04005 [astro-ph.HE]].

[5] V. S. Berezinsky and G. T. Zatsepin, Phys. Lett. B 28 (1969), 423-424 doi:10.1016/03702693(69)90341-4

[6] K. Fang, K. Kotera, K. Murase and A. V. Olinto, Phys. Rev. D 90 (2014) no.10, 103005 [erratum: Phys. Rev. D 92 (2015) no.12, 129901] doi:10.1103/PhysRevD.90.103005 [arXiv:1311.2044 [astro-ph.HE]].

[7] M. Chianese, D. F. G. Fiorillo, R. Hajjar, G. Miele, S. Morisi and N. Saviano, JCAP 05 (2021), 074 doi:10.1088/1475-7516/2021/05/074 [arXiv:2103.03254 [hep-ph]].

[8] J. A. Aguilar et al. [RNO-G], JINST 16 (2021) no.03, P03025 doi:10.1088/17480221/16/03/P03025 [arXiv:2010.12279 [astro-ph.IM]].

[9] M. G. Aartsen et al. [IceCube], [arXiv:1911.02561 [astro-ph.HE]].

[10] M. G. Aartsen et al. [IceCube-Gen2], J. Phys. G 48 (2021) no.6, 060501 doi:10.1088/13616471/abbd48 [arXiv:2008.04323 [astro-ph.HE]].

[11] J. Álvarez-Muñiz et al. [GRAND], Sci. China Phys. Mech. Astron. 63 (2020) no.1, 219501 doi:10.1007/s11433-018-9385-7 [arXiv:1810.09994 [astro-ph.HE]].

[12] J. F. Navarro, C. S. Frenk and S. D. M. White, Astrophys. J. 462 (1996), 563-575 doi:10.1086/177173 [arXiv:astro-ph/9508025 [astro-ph]].

[13] C. W. Bauer, N. L. Rodd and B. R. Webber, JHEP 06 (2021), 121 doi:10.1007/JHEP06(2021)121 [arXiv:2007.15001 [hep-ph]].

[14] N. Aghanim et al. [Planck], Astron. Astrophys. 641 (2020), A6 doi:10.1051/00046361/201833910 [arXiv:1807.06209 [astro-ph.CO]]. 
[15] P. Allison et al. [ARA], Phys. Rev. D 93 (2016) no.8, 082003 doi:10.1103/PhysRevD.93.082003 [arXiv:1507.08991 [astro-ph.HE]].

[16] A. Esmaili, A. Ibarra and O. L. G. Peres, JCAP 11 (2012), 034 doi:10.1088/14757516/2012/11/034 [arXiv:1205.5281 [hep-ph]].

[17] K. Ishiwata, O. Macias, S. Ando and M. Arimoto, JCAP 01 (2020), 003 doi:10.1088/14757516/2020/01/003 [arXiv:1907.11671 [astro-ph.HE]]. 